\section{Research Square}

Preprints are preliminary reports that have not undergone peer review.

They should not be considered conclusive, used to inform clinical practice, or referenced by the media as validated information.

\title{
Quality of Life and Its Correlates Among Clients Visiting Antiretroviral Therapy Clinics in Addis Ababa, Ethiopia
}

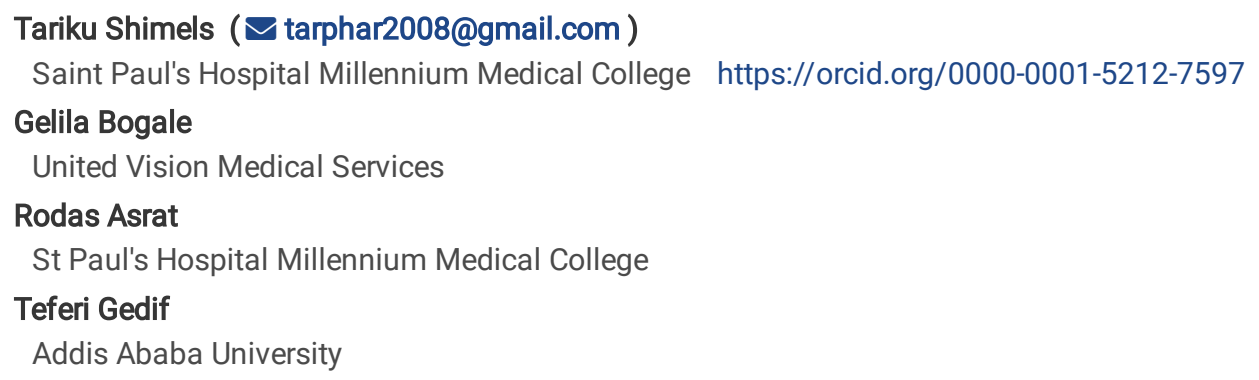




\section{Abstract}

Background: As a chronic illness with potential complications in physical, emotional, social, and economic aspects, HIV poses deterioration in patients' quality of life.The aim of this study was to assess status of the functional and current quality of life, and associated factors among clients visiting ART clinics in Addis Ababa, Ethiopia.

Methods: A multi-site cross-sectional study was conducted in August 2020. All adults with HIV and on active ART at least for 6 months earlier to the study period, and visiting the selected public health facilities were considered. Facilities were selected purposively, and clients were enrolled using a systematic random sampling technique after a proportion to size allocation. Data were collected using a modified and translated questionnaire of the EQ 5D and EQ-VAS. The analysis was done using SPSS v.26 for windows. Descriptive statistics and non-parametric inferential tests namely; Mann Whitney $U$ test, Kruskal Wallis test, and Spearman's correlation tests were carried out to test the presence of correlates with dependent variables. Ethical approval was sought from Saint Paul's Hospital Millennium Medical College and Addis Ababa regional health bureau.

Results: Out of 371 clients, the majority were from health centers (58\%), aged 43 years or below (54\%), males (63\%), either with primary or secondary education (61\%) and married (46.6\%) while half were either government or private employees. Pain/discomfort and depression/anxiety were the dimensions most affected with predominance in the age group above 43. The mean score of EQ 5D index values was 0.87 (SD: $\pm 0.05 ; 95 \%$ C.I; 0.86-0.87) and that of EQ-VAS was 81 (SD: $\pm 15,95 \%$ C.I: $80-83$ ). About $38 \%$ and $43 \%$ of the clients showed an altered health state of any degree in functional and current overall status respectively. Comorbidity, history of current substance use, facility type, social support, and sleep disturbance showed a statistically significant correlation with the functional and current overall health status.

Conclusion: A considerable proportion of clients have altered functional and overall health status with pain/discomfort and depression/anxiety most affected. Inclusion of multidisciplinary treatment for HIV and the highlighting of psychosocial issues that are likely amongst this group are warranted.

\section{Background}

Quality of life (QoL) is a comprehensive, but fundamental dimension to measure the impact of policies and interventions in health studies [1, 2]. The concept closely accords with World Health Organization's (WHO) definition of health as 'state of complete physical, mental and social wellbeing, and not merely the absence of disease and infirmity' [3].And, variations of the subject, such as a state of well-being [4], satisfaction of individual values [5], individuals' perception of their position in life [6], and overall enjoyment of life [7] have been used in earlier literature. However, as there is no single definition for QoL, researchers are advised to specify the facets included in their evaluation [8]. One of the widely applied generic measurements for health utilities, in recent studies, is the European QoL five dimensional (EQ 5D) instrument which comprises of mobility, self-care, usual activity, pain/discomfort and depression/anxiety elements along with a visual analogue scale [9].

As a chronic illness potentially entailing with complications in physical, emotional, social and economic aspects, human immune virus (HIV) poses unquantifiable challenges on patients' QoL $[10,11]$. Even in the presence of combined antiretroviral therapy (ART), QoL in this group has been reported to be lower compared to the general population [12]. This is likely as clients may suffer from perceived background of risk behavior, stigma and discrimination in social affairs [13] suggesting that there are multiple factors other than ART which affect QoL and need to be addressed [14].

The distribution of HIV, across Ethiopia, varies by geographic and socio-demographic attributes. According to a report by the United Nations program on HIV/AIDS (UNAIDS) [15], about 690000 people were living with HIV in 2018 with an adult (15-49 years) prevalence of was 0.9\%. Women accounted the higher percentage (63\%) in this estimation. A projection for 2020 also showed that nearly 745,719 people would be affected by the virus with a $94.5 \%$ proportion among the adult population [16]. Addis Ababa accounted the second highest prevalence of HIV in Ethiopia (3.4\%) next, only, to Gambella region (4.8\%) [17].

Previous studies documented several factors to correlate with QoL of people living with HIV. These included; age, CD4 count, and ART adherence [14]; severity of depression, deterioration of work function, lack of social support, negative stressors, and adverse effects of HAART [18]; social support, self-esteem and sexuality problems [19], as well as lack of healthcare insurance, having no basic knowledge of HIV/AIDs prior to diagnosis, low monthly income of participants and family, and stigma [20] among others.

The negative impact that the COVID-19 pandemic might has posed on the clinical service in general and ART clients in particular cannot, easily, be undermined. One such an effect could be interruption of prevention activities as well as treatment service for both new and existing cases during high health system demand [21, 22]. Fear of acquiring COVID-19 infection, financial problems have limited clients' visit to ART clinics [23]. While the prevalence of mental health problems among people with HIV is reported to be high [24], the COVID-19 pandemic can also worsen this concern leading to affected health state. 
Assessing the QoL of clients receiving ART services may provide a timely update on the picture of health status among this vulnerable group. The application of a combined functional and current overall measurement can also elucidate a comprehensive evidence of perceived health status clients had experienced, notably, amidst of the COVID-19 pandemic. The present study, therefore, aims to assess correlates of functional and current quality of life among clients visiting ART clinics in Addis Ababa, Ethiopia.

\section{Methods}

\section{Study setting, design and period}

This study was conducted in Addis Ababa, the capital city of Ethiopia. The city has a projected population over 3.9 million in 2020 [25]. There are a total of twelve public hospitals in Addis Ababa [26]. Whereas six are administered as federal hospitals under the ministry of health (FMOH), the remaining five hospitals and 103 health centers are administered under the city administration (AAHB) [27]. A cross-sectional study was undertaken in seven public health facilities which provide antiretroviral therapy services to clients. Saint Paul's Hospital Millennium Medical College (SPHMMC), a teaching hospital under the ministry and Ras Desta Damtew Memorial Hospital (RDDMH), a referral hospital under the city government were included. Likely, five health centers namely; Arada, Lideta, Nifas Silk Lafto Wereda 9, Akaki Kality and Bulbula health centers were also selected. The study was conducted in August 2020, five months after the COVID-19 pandemic was declared in Ethiopia.

\section{Population}

The target population is all adult clients diagnosed with HIV and were placed on ART regimens six months prior to the start of this study. The study population was all adult HIV positive clients who visited the selected study sites and fulfilled the inclusion criteria.

\section{Inclusion and exclusion criteria}

Adult clients living with HIV, either visited the selected health facilities themselves or approached by health professionals for refills, placed on either first or second line ART at least six months earlier and had appointments during the study period were eligible. A client was considered ineligible if at least one of these were noted: admitted to any inpatient ward, experienced any form of debilitating mental health problem (s) or not consented to take part in the study.

\section{Sample size and sampling technique}

Since no report, applying the same measurement scale, was available in the current setting, we assumed the level of affected quality of life both for functional and current overall health status as $50 \%$. The single population proportion formula was employed with $95 \%$ confidence level and $5 \%$ precision producing up a required sample size of 385 . Health facilities were selected purposively based on flow of clients and representation. Allocation to respective health facilities was done using a proportion to size ratio. A systematic random sampling technique was used to select clients. The ART registry book was used as a sampling frame. Participants who had appointments during the study period but were unable to attend health facilities were contacted at their residence home.

\section{Data collection questionnaire, procedure and quality assurance}

Data on client profiles, clinical and quality of life measurement was collected using a pre-tested Amharic version of structured questionnaire. Clients' QoL was assessed by using the EQ-5D-3L instrument [28] which has five dimensions namely: mobility, self-care, usual activities, pain/discomfort and depression/anxiety on three levels as; level I, II and III. Each level corresponds to functional health status of no problems, some or moderate problems and severe/extreme problems. Likely, patients' subjective judgement about their current state of overall health was measured using the Euro QoL visual analogue scale (EQ VAS) [28] presented as a simplified version [29]. Data collectors were trained ahead of deployment. Reliability of the instrument was checked using the Cronbach's alpha test. The study team revised the validity of the tool to contain comprehensive data. Supervision of the data collection process was performed on a continuous basis.

\section{Variables of the study}

The dependent variable was clients' functional and current overall health status. The independent variables included: age, sex, marital status, income level, number of families, occupation, substance use, presence of social support and education level, type of health facility, presence of sleep disturbance problem, presence of comorbid condition, duration of disease, and medication profiles.

\section{Data analysis}

Collected data was cleaned manually, coded and entered in SPSS version 26.0. Exploratory data analysis was done to check for coding errors and missed entries. Index values for QoL were estimated using the health state valuation weights in Zimbabwe [30]. Descriptive statistics, using univariate and bivariate analysis, was applied to present EQ 5D profiles as mean, standard deviation, frequencies and percentages. Spearman's correlation test was used to test presence of correlation between dependent and independent variables. Normality of EQ 5D and EQ VAS scores 
was tested by Shapiro-Wilk test. Non-parametric models namely; Mann Whitney U test and Kruskal Wallis tests were used to assess correlates of outcome scores as ranked mean. A p-value of 0.05 was considered as a cut point of statistical significance in all difference tests.

\section{Ethical approval}

Ethics consideration was sought at two institutions; Saint Paul's hospital millennium medical college (SPHMMC) institution review board and the Addis Ababa Health Bureau (AAHB) research ethics committee, which both are available with the corresponding author. Further, a support letter was written to the respective facilities. Because participation in the study has less than minimal risk, an informed verbal consent was obtained from all participants. Respondents were aware that there was no direct benefit obtained by taking part in the study, and that findings would help to recommend for further holistic approaches along with ART. Participation in the study was entirely on a voluntarily basis. Data acquired was kept confidential throughout and after completion of the study.

\section{Operational definitions}

Affected or unaffected functional health status: computed from a transformed EQ 5D rating at three levels, an index value set at or below the mean (0.87) was considered as affected whilst a score above it was regarded unaffected.

Affected or unaffected current (overall) health status: reflects clients' self-rating of their own current overall health status on a numerical EQ VAS scale ranging from 0 (worst imaginable health state) to 100 (best imaginable health state). A score at or below the mean (81) was considered affected while scores beyond accounted unaffected health status.

Current history of any substance use: implies to client's report of using either of Khat (Catha edulis), alcohol, tobacco, other substances or any combination in the past three months.

\section{Results}

\section{Socio-demographic profile of respondents}

A total of 371 participant questionnaires were complete, and considered for a subsequent analysis. Mean (SD) of age was included in the study, 215 (58\%) were from health centers, 200 (54\%) were aged 43 years or below, 233(62.8\%) were males, 225(60.6\%) were either with primary or secondary education, 262(70.6\%) were Orthodox Christian, 186(50\%) were either government or private employees, 173(46.6\%) were married and $343(92.5 \%)$ do not have a current history of any substance use (table 1 ).

Table 1: Socio-demographic profiles of respondents, Addis Ababa, Ethiopia, August 2020 ( $n=371)$ 


\begin{tabular}{|c|c|c|c|}
\hline Variable & Label & Frequency & Percent \\
\hline \multirow[t]{2}{*}{ Facility } & Health center & 215 & 58.0 \\
\hline & Hospital & 156 & 42.0 \\
\hline \multicolumn{4}{|c|}{ Age $(\text { Yrs. })^{a}$} \\
\hline & $=<43$ & 200 & 53.9 \\
\hline & $>43$ & 171 & 46.1 \\
\hline \multicolumn{4}{|l|}{ Sex } \\
\hline & Male & 138 & 37.2 \\
\hline & Female & 233 & 62.8 \\
\hline \multicolumn{4}{|c|}{ Education level } \\
\hline & Not/able to read and write & 87 & 23.5 \\
\hline & Primary/high school education & 225 & 60.6 \\
\hline & College/university education & 59 & 15.9 \\
\hline \multicolumn{4}{|l|}{ Religion } \\
\hline & Orthodox Christian & 262 & 70.6 \\
\hline & Protestant Christian & 57 & 15.4 \\
\hline & Muslim & 46 & 12.4 \\
\hline & Others ${ }^{b}$ & 6 & 1.6 \\
\hline \multicolumn{4}{|c|}{ Occupation } \\
\hline & Merchant & 17 & 4.6 \\
\hline & Government / private employee & 186 & 50.1 \\
\hline & Student & 17 & 4.6 \\
\hline & Housewife/jobless & 119 & 32.1 \\
\hline & Others ${ }^{c}$ & 32 & 8.6 \\
\hline \multicolumn{4}{|c|}{ Marital status } \\
\hline & Unmarried & 89 & 24.0 \\
\hline & Married & 173 & 46.6 \\
\hline & Widowed/separated & 109 & 29.4 \\
\hline \multicolumn{4}{|c|}{ Current substance use history } \\
\hline & No & 343 & 92.5 \\
\hline & Yes & 28 & 7.5 \\
\hline
\end{tabular}

Remark: ${ }^{a}$ categorized based on mean age of respondents; ${ }^{b}$ indicates: Catholic, Waqe feta and Jehovah witness; ${ }^{c}$ indicates: prostitutes, daily laborers and 'safety net' aid receivers.

\section{EQ-5D-3L functional health states}

The descriptive presentation of EQ 5D items illustrate that majority of the clients had no problems. Yet, an increasing trend of affected health dimension was noted as moderate problems persist in self-care $(15,4 \%)$, usual activity $(18,4.9 \%)$, mobility $(36,9.7 \%)$, pain/discomfort $(75$, $20.2 \%)$ and depression /anxiety $(89,24 \%)$. Severe/extreme problems were apparent for pain/discomfort and depression/anxiety dimensions reported by $4(1.1 \%)$ and $10(2.7 \%)$ of the clients respectively (figure 1$)$. 
When an age-wise distribution of EQ 5D elements were assessed, level II to level III problems were more pronounced in the age group beyond 43 for mobility, self-care and usual activities. Frequent reports were noted on pain/discomfort (21.2\%) and depression/anxiety (23.2\%) in the lower age group. These dimensions have also accounted a relatively higher percentage of severe problems in the late age group (table 2 ).

Table 2: Age-wise classification of EQ 5D items among clients on ART in Addis Ababa, August, 2020

\begin{tabular}{|c|c|c|c|c|c|c|}
\hline \multirow[t]{2}{*}{ Age category (yrs.) } & & \multicolumn{5}{|c|}{ EQ-5D-3L scale } \\
\hline & & $\begin{array}{l}\text { Mobility } \\
\text { n (\%) }\end{array}$ & $\begin{array}{l}\text { Self-care } \\
\text { n (\%) }\end{array}$ & $\begin{array}{l}\text { Usual activities } \\
\text { n (\%) }\end{array}$ & $\begin{array}{l}\text { Pain/ discomfort } \\
\text { n (\%) }\end{array}$ & $\begin{array}{l}\text { Depression/ } \\
\text { anxiety } \\
\text { n (\%) }\end{array}$ \\
\hline \multirow{3}{*}{$\begin{array}{l}<=43 \\
(n=203)\end{array}$} & Level 1 & 188(92.6) & 197(97) & 196(96.6) & 159(78.3) & $147(72.4)$ \\
\hline & Level 2 & $15(7.4)$ & $6(3)$ & $7(3.4)$ & $43(21.2)$ & $47(23.2)$ \\
\hline & Level 3 & $0(0)$ & $0(0)$ & $0(0)$ & $1(0.5)$ & $9(4.4)$ \\
\hline \multirow{3}{*}{$\begin{array}{l}>43 \\
(n=168)\end{array}$} & Level 1 & $146(86.9)$ & 158(94) & 156(92.9) & $133(79.2)$ & $125(74.4)$ \\
\hline & Level 2 & $21(12.5)$ & $9(5.4)$ & $11(6.5)$ & $32(19)$ & $42(25)$ \\
\hline & Level 3 & $1(0.6)$ & $1(0.6)$ & $1(0.6)$ & $3(1.8)$ & $21(0.6)$ \\
\hline
\end{tabular}

Remark: Level I=no problems, level II=some/moderate problems, level III=severe/extreme problems

\section{Functional and current health status}

A functional and current overall health status was derived from EQ 5D-3L and EQ VAS scores respectively. The mean (SD) score of EQ 5D index values was $0.87( \pm 0.05)$ ranging from 0.64 to 0.90 . And, mean (SD) of EQ VAS was $81( \pm 15)$ ranging from 40 to 100 . About 230 (62\%) of the respondents scored an index value above the mean for functional health states on the EQ 5D dimensions whilst 213 (57.4\%) scored above the mean for current overall health status (figure 2).

\section{Correlates of functional and current health status}

Multiple predictors have been tested for potential association with both functional and current overall quality of life. These two outcome variables, with a statistically significant positive correlation $(r=0.5, p=0.000)$, reflect the functional and current overall health rating of clients respectively. Age, income and duration of disease did not show any correlation with both outcomes using the spearman's test. The outcome variables showed a non-normal distribution hence, necessitating options in non-parametric statistics. Accordingly, the Kruskal Wallis test showed that no associations exist between the dependent variables versus education level, marital status and occupation of clients.

Meanwhile, a Mann Whitney test revealed that mean rank of EQ 5D and EQ VAS scores were higher among clients with no comorbidity, no sleep disturbance and those visiting health centers. A higher rank of EQ 5D index value, but not EQ VAS, score was noted among clients with least/neutral to issues for getting help from others and those with 8 years or less duration on ART medication. In the contrary, only EQ VAS score was higher among clients with no current history of any substance use (table 3 ).

Table 3: Correlates of functional and current overall health status among clients on ART in Addis Ababa, Ethiopia, August 2020 ( $n=371$ ) 


\begin{tabular}{|c|c|c|c|c|c|c|c|c|}
\hline \multirow[t]{2}{*}{ Characteristics } & & \multirow[b]{2}{*}{$\mathbf{N}$} & \multicolumn{3}{|c|}{ EQ 5D index values $(0.4-0.9)$} & \multicolumn{3}{|c|}{ EQ VAS scores $(40-100)$} \\
\hline & & & Mean rank & $\mathrm{U}$ & P-value & Mean rank & $\mathbf{U}$ & P-value \\
\hline \multicolumn{9}{|c|}{ Comorbid condition } \\
\hline & No & 282 & 198.6 & \multirow[t]{2}{*}{8997.0} & \multirow[t]{2}{*}{$0.000^{\star}$} & 200.12 & \multirow[t]{2}{*}{8568.0} & \multirow[t]{2}{*}{$0.000^{\star}$} \\
\hline & Yes & 89 & 146.1 & & & 141.27 & & \\
\hline \multicolumn{9}{|c|}{ Current history of any substance use } \\
\hline & No & 343 & 185.99 & \multirow[t]{2}{*}{4806.0} & \multirow[t]{2}{*}{0.993} & 190.47 & \multirow[t]{2}{*}{3267.5} & \multirow[t]{2}{*}{$0.004^{\star}$} \\
\hline & Yes & 28 & 186.14 & & & 131.20 & & \\
\hline \multicolumn{9}{|c|}{ Presence of sleep disturbance } \\
\hline & No & 293 & 199.51 & \multirow[t]{2}{*}{15385.0} & \multirow[t]{2}{*}{$0.000^{\star}$} & 195.75 & \multirow[t]{2}{*}{14284.0} & \multirow[t]{2}{*}{$0.001 *$} \\
\hline & Yes & 78 & 135.56 & & & 149.37 & & \\
\hline \multicolumn{9}{|l|}{ Facility type } \\
\hline & Health center & 215 & 203.35 & \multirow[t]{2}{*}{13039.00} & $0.000^{\star}$ & 219.07 & \multirow[t]{2}{*}{9660.5} & \multirow[t]{2}{*}{$0.000 *$} \\
\hline & Hospital & 156 & 162.08 & & & 140.43 & & \\
\hline \multicolumn{9}{|c|}{ Getting help from others } \\
\hline & Difficult & 158 & 155.05 & \multirow[t]{2}{*}{21716.5} & \multirow[t]{2}{*}{$0.000^{\star}$} & 179.21 & \multirow[t]{2}{*}{17900.0} & \multirow[t]{2}{*}{0.286} \\
\hline & Neutral/easy & 213 & 208.96 & & & 191.04 & & \\
\hline \multicolumn{9}{|c|}{ ART duration (Yrs.) } \\
\hline & $<=8$ & 213 & 194.46 & \multirow[t]{2}{*}{15025.0} & \multirow[t]{2}{*}{$0.043^{\star}$} & 180.20 & \multirow[t]{2}{*}{18062.0} & \multirow[t]{2}{*}{0.219} \\
\hline & $>8$ & 158 & 174.59 & & & 193.82 & & \\
\hline
\end{tabular}

*Indicates a significance level at $\mathrm{P}<0.05$.

\section{Discussion}

Previous studies have indicated that quality of life was reported to be affected prominently in people living with HIV [10, 11]. This study was conducted to uncover any potential areas of increased impact during the COVID-19 pandemic as it has compromised clinical services and might have presented a barrier to obtaining health services by posing a health threat. As a report also suggested that quality of life is not restricted to the use of ART [12], other issues around quality of life need to be looked at in this vulnerable group.

When investigating functional health status of clients, moderate alterations persisted to all dimensions whereas, severe forms of affected health dominate in pain /discomfort and depression /anxiety scores. And, adjusted for age, moderate problems in self-care, mobility and daily activities were found in those 43 years and beyond; while the same measurement in pain/discomfort and depression/anxiety were experienced across all ages, but predominantly witnessed above the 43 years group. Encounter of poor outcomes in these dimensions has, elsewhere, been reported $[31,32]$.

The moderate problem in self-care, mobility and daily activities could be accounted for the early occurrence of comorbidities in people living with HIV in the developing countries because both EQ-5D and EQ VAS are found to be better among clients who do not have co-morbidities. A case control study conducted in Brazil showed that people living with HIV had similar age related comorbidities but 10 years earlier [33]. However in contrast to this, our study doesn't show significant relationships between occurrence of comorbidities and duration of illness (mean rank $=180.23$ vs $204.3, p=0.064$ ). This can be explained by the fact that the earlier study considered a population group aged beyond 50 years (median=57). It can also be accounted for by the fact that co-morbidities tend to be prevalent in people with HIV as having HIV coupled with the ART may predispose them to co-morbidities [34].

Result of this study confirmed that clients have a comparable rating in current state of overall quality of life as majority had similar value sets to the general population of the Zimbabwean context [30] with mean (SD) of EQ VAS ratings was 81 (95\% C.l; 79.8-83). Yet, the mean of EQ-5D $(0.87,95 \%$ C.l; $0.86-0.87)$ is above the reference population average which might be attributed to other socio-economic and individual variations. Despite a need for cautious interpretation of findings, the reliability coefficient of the scale was within the acceptable range (Cronbach's alpha $=0.70$ ). Based on a dichotomous classification of the respective QoL measurements, it was noted that $38 \%$ were affected at any degree of 
functional status while $43 \%$ rated themselves as affected with current health state. Whilst differences could exist as current health status was estimated from mean of EQ VAS scores, it is worth noting that both the EQ 5D and EQ VAS scales showed a significant positive correlation $(r=0.5, p=0.000)$.

Although male participants take the majority of the data set (62.8\%), there has not been any difference in the measured functionality or current health status by sex. Despite the pre-assumption of the study that the COVID-19 pandemic might have compromised health seeking behavior by imposing a financial burden, no significant difference in functionality or overall health status was found among clients with different occupations and income brackets.

We also found that those clients with no sleep disturbance $(p=0.000)$ were found to have better functionality and overall current health status. Altered sleep pattern is one of the criteria for major depressive disorder on diagnostic statistical manual - V (DSM-V) and the finding of good sleep pattern can be a reflection of good mental health status and improved functionality. While its absence mediates depression [35], good sleep can also improve bodily pain and vitality [36]. It has also been found that presence of depression and the severity of depression were related with the quality of life [18].

In this study, clients with a follow up at health centers were found to have better indicators of functionality and overall health status. This could be explained by a reduced number of catchment population utilizing ART services as compared to the hospitals. It can also be due to a more focused area of services offered at health centers as compared to the varied services provided at hospitals.

In the same pattern, it is evident that a decrease in current history of substance use is associated with a better current overall health status. The questionnaire used for the study assessed the presence of history in current use of alcohol, chat (amphetamine related substance commonly used in Ethiopia) and smoking. The relationship of substance abuse and its impact on health related quality of life has been discussed in previous studies especially that of sedatives and amphetamines [37]. This goes in line with the finding of our study and further solidifies the indication that improved mental health is associated with better functionality and overall health status.

In spite of lacking many comparative studies which measured QoL of ART clients with the same scale, this study has tried to present a current update on the issue by applying the discriminant and generic EQ 5D and EQ VAS instrument. Inclusion of more facilities may preset advantages to contain client diversity and representation of reports. However, the study used an index value reported from Zimbabwe [30] as there is no standardized, country specific value set of EQ 5D scale in Ethiopia.

\section{Conclusion}

It is evident from the study that the health related quality of life of clients was found to be lower in those aged 43 and above. The finding also revealed that psychosocial factors are detrimental for the health related quality of life of people living with HIV. A considerable proportion of clients have affected functional and current overall health status. Comorbid condition, history of current substance use, facility type and sleep disturbance showed a statistically significant correlation with QoL of clients. Overall, the study found that there is a need for continuous surveillance of the health related quality of life among people living with HIV. The authors suggest for the inclusion of a multidisciplinary treatment for HIV and the highlighting of psychosocial issues that are likely amongst this group.

\section{List Of Abbreviations}

AAHB, Addis Ababa Health Bureau; AIDS, Acquired immune Deficiency Syndrome; ART, Anti-Retroviral Therapy; COVID-19, Coronavirus Disease 2019; DSM-V, Diagnostic Statistical Manual-V; EQ 5D 3L, EuroQol 5 Dimension 3 Level; EQ VAS, Euro Qol Visual Analogue Scale; FMOH, Federal Ministry of Health; HAART, Highly Active Anti-Retroviral Therapy; HIV, Human Immune Virus; RDDMH, Ras Desta Damtew Memorial Hospital; SPHMMC, Saint Paul's Hospital Millennium Medical College; WHO, World Health Organization.

\section{Declarations}

\section{Ethics Approval and Consent}

Ethics consideration was sought at two institutions; Saint Paul's hospital millennium medical college (SPHMMC) institution review board and the Addis Ababa Health Bureau (AAHB) research ethics committee, which both are available with the corresponding author. Further, a support letter was written to the respective facilities. Because participation in the study has less than minimal risk, an informed verbal consent was obtained from all participants. Respondents were aware that there was no direct benefit obtained by taking part in the study, and that findings would help to recommend for further holistic approaches along with ART. Participation in the study was entirely on a voluntarily basis. Data acquired was kept confidential throughout and after completion of the study.

\section{Consent for publication}

Page $8 / 11$ 
Not applicable.

\section{Availability of data}

The datasets analyzed during the current study are available from the corresponding author.

\section{Competing interests}

The authors declare that they have no competing interests.

\section{Funding}

Funding for this study was obtained from Saint Paul's Hospital Millennium Medical College.

\section{Author Contributions}

All authors have made substantial contributions to conception and design, acquisition of data, or analysis and interpretation of data; drafting the article or revising it critically, and to submit to the current journal, gave approval of the version to be published; and agree to be accountable for all aspects of the work.

\section{Acknowledgments}

The authors would like to thank the clients who provided their informed consent, and participated in this study. Authors would also like to thank concerned department coordinators in the respective facilities, and the data collectors.

\section{References}

1. G. A. Gellert. The Importance of Quality of Life Research for Health Care Reform in the USA and the Future of Public Health. Quality of Life Research1993; 2, (5): pp. 357-361. JSTOR, www.jstor.org/stable/4034761. Accessed 22 Nov. 2020.

2. Buiting H.M and Olthuis G. Importance of Quality-of-Life Measurement throughout the Disease Course. JAMA Netw Open 2020;3(3):e200388. doi:10.1001/jamanetworkopen.2020.0388. Accessed on 22 November 2020.

3. CONSTITUTION of the World Health Organization. Chron World Health Organ 1947; 1(1-2):29-43.

4. Gotay CC, Moore TD. Review Assessing quality of life in head and neck cancer. Qual Life Res 1992 Feb; 1(1):5-17.

5. Emerson EB. Evaluating the impact of deinstitutionalization on the lives of mentally retarded people. Am J Ment Defic 1985; 90(3):277-88.

6. The WHOQOL Group. Development of the World Health Organization WHOQOL-BREF quality of life assessment. Psychol Med 1998 May; 28(3):551-8.

7. Rush AJ, South CC, Jha MK, Grannemann BD, Trivedi MH. Toward a very brief quality of life enjoyment and Satisfaction Questionnaire. Journal of Affective Disorders 2019;Volume 242: Pages 87-95. Available on: https://doi.org/10.1016/j.jad.2018.08.052. Accessed on 9 December 2020.

8. Post MW. Definitions of quality of life: what has happened and how to move on. Top Spinal Cord Inj Rehabil 2014;20(3):167-180. doi:10.1310/sci2003-167.

9. EuroQol research foundation. EQ-5D user guide 2018. Available at; http//:www.euroqol.org/publications/user.guide

10. Balderson BH, Grothaus L, Harrison RG, McCoy K, Mahoney C, Catz S. Chronic illness burden and quality of life in an aging HIV population. AIDS Care 2013;25(4):451-458. doi:10.1080/09540121.2012.712669

11. Basavaraj KH, Navya MA, Rashmi R. Quality of life in HIV/AIDS. Indian J Sex Transm Dis AIDS 2010; 31(2):75-80. doi:10.4103/02537184.74971

12. Miners A, Phillips A, Kreif N, Rodger A, Speakman A, Fisher M et al. Health-related quality-of-life of people with HIV in the era of combination antiretroviral treatment: a cross-sectional comparison with the general population. The Lancet HIV 2014; 1(1); Pages e32-e40. Available at: https://doi.org/10.1016/S2352-3018(14)70018-9. Accessed on 3 December 2020.

13. Saki M, Khan M, Kermanshahi S, Mohammadi E, Mohraz M. Perception of Patients With HIV/AIDS From Stigma and Discrimination. Iran Red Crescent Med J 2015;17(6):e23638. Published 2015 Jun 23. doi:10.5812/ircmj.23638v2

14. Liping M, Peng X, Haijiang L, Lahong J, Fan L. Quality of Life of People Living with HIV/AIDS: A Cross-Sectional Study in Zhejiang Province, China. PLoS ONE 2015; 10(8): e0135705. https://doi.org/10.1371/journal.pone.0135705

15. UNAIDS. Ethiopia/UNAIDS. Available at: https://www.unaids.org/en/regionscountries/countries/ethiopia\#: :text=In\%20Ethiopia\%20in\%202018\%3A,49\%20years)\%20was\%201\%25. Accessed on 22 November 2020. 
16. Ethiopian Public Health Institute (EPHI). HIV Related Estimates and Projections for Ethiopia. Addis Ababa. Ethiopia. EPHI Available at: https://www.ephi.gov.et/images/pictures/download2009/HIV_estimation_and_projection_for_Ethiopia_2017.pdf Accessed on 22 November 2020.

17. HAPCO. HIV prevention in Ethiopia National Road map; 2018-2020. HAPCO 2018. Available at: https://ethiopia.unfpa.org/sites/default/files/pub-pdf/HIV\%20Prevention\%20in\%20Ethiopia\%20National\%20Road\%20Map\%202018\%20\%202020\%20FINAL_FINAL.pdf accessed on 22 November2020.

18. Yen CF, Tsai JJ, Lu PL, Chen YH, Chen TC, Chen PP, et al. Quality of life and its correlates in HIV/AIDS male outpatients receiving highly active antiretroviral therapy in Taiwan. Psychiatry Clin Neurosci 2004;58:501-506. https://doi.org/10.1111/j.1440-1819.2004.01292.x

19. den Daas, C, van den Berk, G.E.L., Kleene, M.J.T. E. S. de Munnik, J. G. Lijmer \& K. Brinkman. Health-related quality of life among adult HIV positive patients: assessing comprehensive themes and interrelated associations. Qual Life Res 2019; 28, 2685-2694. https://doi.org/10.1007/s11136-019-02203-y

20. Ebrahimi Kalan M, Han J, Ben Taleb Z, Fennie KP, Asghari Jafarabadi M, Dastoorpoor M, Hajhashemi N, Naseh M, Rimaz S. Quality Of Life And Stigma Among People Living With HIV/AIDS In Iran. HIV AIDS (Auckl) 2019;11:287-298 https://doi.org/10.2147/HIV.S221512

21. Lesosky M and Myer L. Modelling the impact of COVID-19 on HIV. The Lancet HIV2020; 7 (9), E596-E598. DOI: https://doi.org/10.1016/S2352-3018(20)30228-9.

22. Hogan AB, Jewell BL, Sherrard-Smith E, Vesga JF, Watson OJ, Whittaker C et al. Potential impact of the COVID-19 pandemic on HIV, tuberculosis, and malaria in low-income and middle-income countries: a modelling study. The Lancet Global Health 2020;8 (9) E1132E1141. DOI: https://doi.org/10.1016/S2214-109X(20)30288-6

23. M. Ponticiello, J. Mwanga-Amumpaire, P. Tushemereirwe, G. Nuwagaba, R. King, R. Sundararajan "Everything is a mess": how COVID-19 is impacting engagement with HIV testing Services in rural southwestern Uganda. AIDS Behav 2020,1-4; 10.1007/s10461-020-02935-w

24. World Health Organization. Mental health and psychosocial considerations during the COVID-19 outbreak. WHO Available at: https://apps.who.int/iris/bitstream/handle/10665/331490/WHO-2019-nCoV-MentalHealth-2020.1-eng.pdf Accessed on 22 November 2020.

25. United Human Settlements Program. The state of Addis Ababa. UN-Habitat 2017; 18-21.

26. Misganaw, A., Mariam, D.H., Araya, T. et al. Patterns of mortality in public and private hospitals of Addis Ababa, Ethiopia. BMC Public Health 2012; 12, 1007. https://doi.org/10.1186/1471-2458-12-1007

27. Bekele GE, Tadesse T, Negaw R, et al. Magnitude and associated factors of hypertension in Addis Ababa public health facilities, Ethiopia. MOJ Public Health 2018;7(6):280-286. http://www.DOI:10.15406/mojph.2018.07.00252

28. EuroQol research foundation. EQ-5D user guide. EuroQo/ Group 2018. Available at; http//:www.euroqol.org/publications/user guide

29. Hyland, M. E. \& Sodergren, S. C. Which global quality of life scale is most reliable and most preferred? Quality of Life Research 1997: 6 (Abstracts: 4th Annual Conference of ISOQOL, 167); 662-63.

30. Jelsma J, Hansen K, De Weerdt W, De Cock P, Kind P. How do Zimbabweans value health states? Popul Health Metr 2003 16;1(1):11. doi: 10.1186/1478-7954-1-11. PMID: 14678566; PMCID: PMC317383.

31. Keaei M, Kuhlmann J, Conde R, Evers SM, Gonzalez J, Govers M et al. Health-Related Quality of Life of Patients with HIV/AIDS in Bogotá, Colombia. Value in health regional issues 2016; 11C;68 - 72.

32. Tran BX, Ohinmaa A, Nguyen LT. Quality of life profile and psychometric properties of the EQ-5D-5L in HIV/AIDS patients. Health Qual Life Outcomes 2012;10:132. Published 2012 Nov 1. doi:10.1186/1477-7525-10-132

33. Maciel RA, Klück HM, Durand M, Sprinz E. Comorbidity is more common and occurs earlier in persons living with HIV than in HIV-uninfected matched controls, aged 50 years and older: A cross-sectional study. Int J Infect Dis 2018;70:30-35. 10.1016/j.ijid.2018.02.009 https://www.sciencedirect.com/science/article/pii/S1201971218300456

34. Getahun Z, Azage M, Abuhay T, Abebe F. Comorbidity of HIV, hypertension, and diabetes and associated factors among people receiving antiretroviral therapy in Bahir Dar city, Ethiopia. J Comorb. 2020 2020; 10. Available at: https://journals.sagepub.com/doi/full/10.1177/2235042X19899319 accessed on 3 December 2020.

35. Becker NB, de Jesus SN, Viseu JN, Stobäus CD, Guerreiro M, Domingues RB. Depression and quality of life in older adults: Mediation effect of sleep quality, International Journal of Clinical and Health Psychology 2018, 18(1): 8-17. https://doi.org/10.1016/j.ijchp.2017.10.002.

36. Lo CM, Lee PH. Prevalence and impacts of poor sleep on quality of life and associated factors of good sleepers in a sample of older Chinese adults. Health Qual Life Outcomes 2012;10:72. Published 2012 Jun 18. doi:10.1186/1477-7525-10-72.

37. Korthuis, P. T., Zephyrin, L. C., Fleishman, J. A., Saha, S., Josephs, J. S., McGrath, M. M., et al. Health-related quality of life in HIV-infected patients: the role of substance use. AIDS patient care and STDs 2008; 22(11), 859-867. https://doi.org/10.1089/apc.2008.0005

\section{Figures}




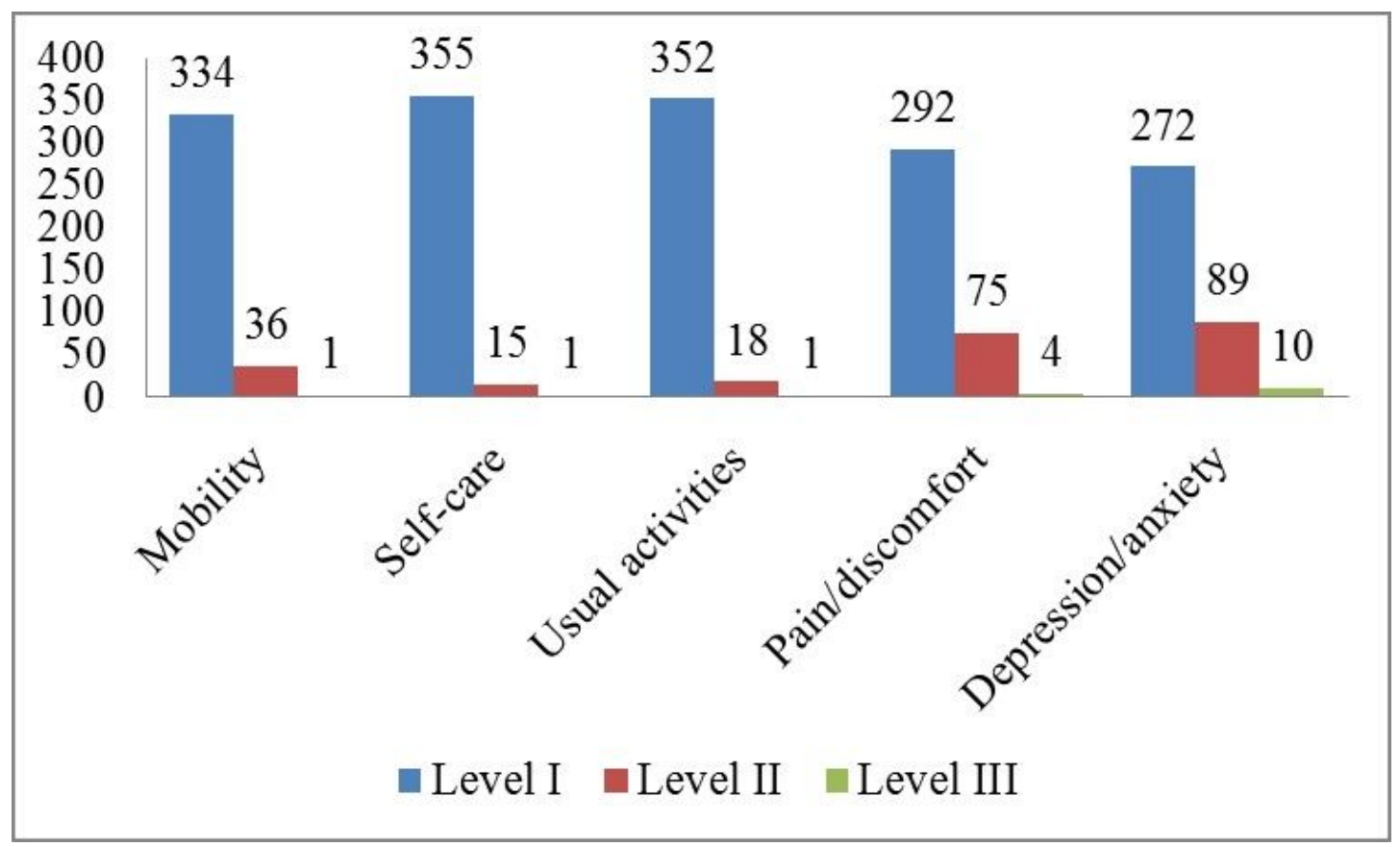

\section{Figure 1}

Descriptive presentation of EQ-5D-3L functional health states among clients on ART in Addis Ababa, Ethiopia, August 2020 ( $n=371$ ) Remark: level I indicates no problems; level II indicates some /moderate problems and; Level III refers severe /extreme problems

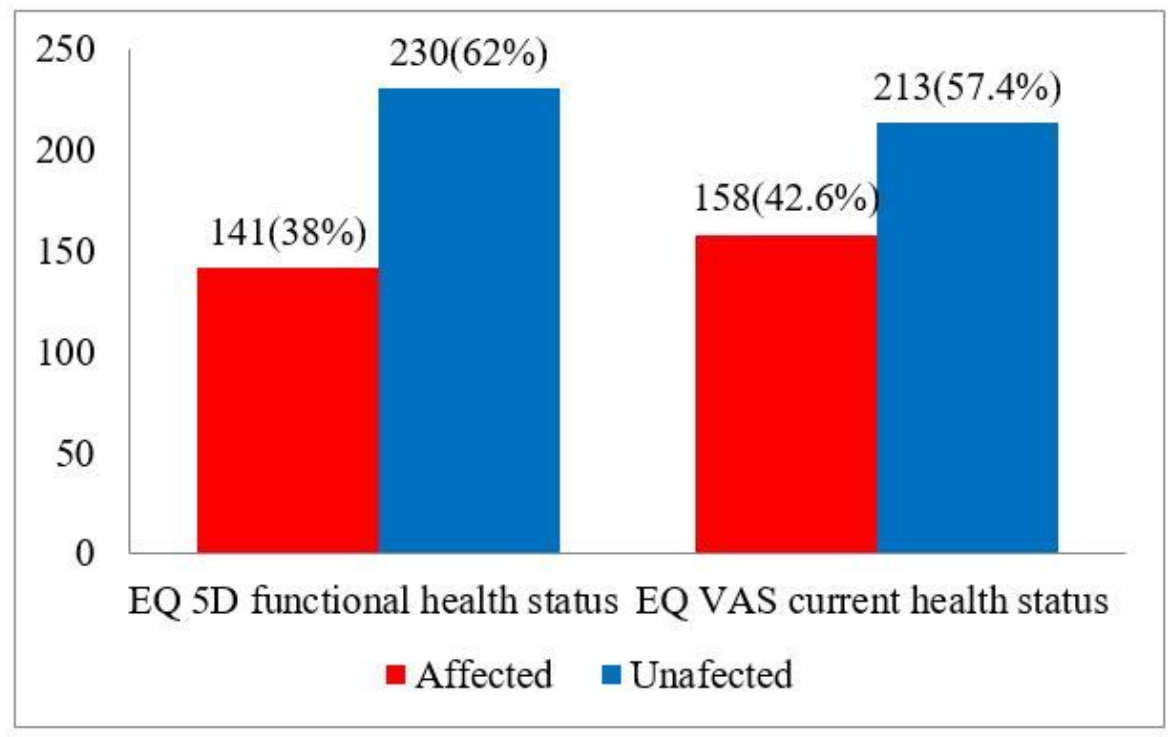

\section{Figure 2}

level of current and functional health status among clients on ART in Addis Ababa, Ethiopia, August 2020 ( $n=371$ ) 